\title{
La variación y el cambio a lo largo de la vida. Entrevista completa a William Labov y Gillian Sankoff
}

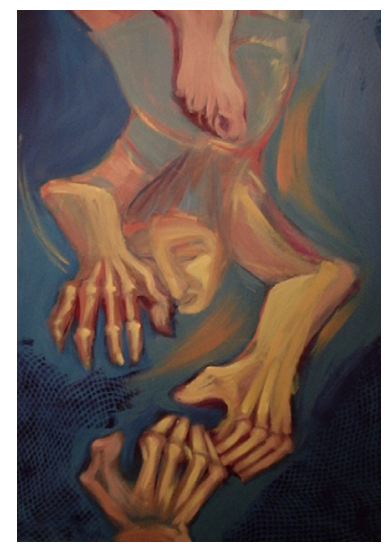

Andrea Menegotto (entrevista, traducción y notas)

Universidad Nacional de Mar del Plata- CONICET, Argentina

acmenegotto@gmail.com

Con la colaboración de Julián Ezquerra

FFyL-Universidad de Buenos Aires, Argentina

julianezquerra@gmail.com

William Labov y Gillian Sankoff estuvieron en Buenos Aires en agosto de 2013 como invitados especiales en las II Jornadas Beatriz Lavandera. La entrevista se realizó el último día del congreso en el mismo Teatro Municipal Gral. San Martín, acompañados por Julián Ezquerra como intérprete en caso de que nos malentendiéramos.

Andrea Menegotto (AM): Quisiera comenzar esta entrevista a partir del trabajo que presentó en su conferencia inaugural ${ }^{1}$, poniendo en funcionamiento esa capacidad humana fundamental de transmitir la experiencia de una persona a otra a través de las narrativas orales de experiencia personal. Quiero pedirles que me cuenten su historia como lingüistas. Su propia narrativa, su experiencia personal como lingüistas.

Gillian Sankoff (GS): Ah, esa es una linda pregunta.

\section{AM: ¿Quién quiere empezar?}

Gillian Sankoff (GS): Bueno, ninguno de los dos empezó por la Lingüística. Tal vez ya sepas que Bill comenzó trabajando como químico.

AM: Sí, ¿trabajaba en algo relacionado con la impresión y las copias, no? GS: Sí, fue el iniciador de un cierto número de fórmulas de tinta para serigrafía... bueno, él te va a contar eso. Yo estudié antropología en la Universidad McGill en Canadá, mi doctorado es en antropología, pero siempre me interesó la lingüística. Como en Montreal no había ningún departamento de lingüística, solo pude tomar algunos pocos cursos de lingüística en distintos departamentos mientras hacía mi carrera de grado y luego mi doctorado en antropología. Mi tema de investigación era el multilinguismo

* Una versión reducida de esta entrevista fue publicada en 2014 en Estudios de teoría literaria. Revista digital de la Facultad de Humanidades de la Universidad Nacional de Mar del Plata. Vol.3 n5: p359 - 366. ISSN 2313-9676 (http://fh.mdp. edu.ar/revistas/index.php/etl/article/view/885). En esa ocasión, habíamos eliminado las cuestiones más técnicas de las que habíamos conversado. Aprovechamos este número especial de Signo y Seña para publicar la versión completa.

1. Labov presentó el análisis de tres narrativas diferentes en inglés y mostró cómo el hablante transforma la realidad asignando elogios y culpas. Recordó a su discípula Beatriz Lavandera con afecto y comentó con anécdotas cómo su "carácter leonino" la condujo en una dirección de resistencia política pero también académica. Vinculó los aportes de Lavandera al análisis del discurso con su propio análisis de las narrativas, particularmente con los dispositivos lingüísticos que utiliza el hablante para polarizar o integrar a los participantes de la historia, y tuvo un especial reconocimiento para los "cachorros" de Lavandera que siguieron la dirección de su trabajo con el discurso político, Salvio Menéndez, Alejandro Raiter y María Marta García Negroni. 
en Papúa Nueva Guinea y ahí fui para recolectar los datos para mi tesis de PhD. Por suerte mi supervisor era una persona muy abierta y me permitió trabajar en lo que yo quisiera, y terminé en un pueblo donde se usaban tres lenguas: la lengua originaria -una lengua nativa austronesia que se llama papua- el tok pisin, - un creole de base inglés derivado de un pidgin y, además, una tercera lengua que era la lengua de los misioneros. Eso fue mi tesis doctoral ${ }^{2}$. Siempre me interesó el multilingüismo porque crecí en Montreal. Yo hablaba inglés, pero éramos una minoría... la mayoría hablaba francés, y el bilingüismo no era tan habitual. Que yo hablara francés fue una especie de shock para la gente que me rodeaba, eso era muy raro en ese momento, pero yo no soportaba no poder hablar con todo el mundo así que aprendí francés. Para mi doctorado me interesó estudiar el fenómeno en un contexto totalmente diferente, y terminé estudiando el multilingüismo en Papua Nueva Guinea. Después de eso conseguí trabajo en un departamento de antropología en la Universidad de Montreal, una universidad de habla francesa. Ahí trabajé once años. Era la "etnolingüista" del departamento de antropología. Me dejaban hacer lo que quisiera, porque en ese momento nadie sabía bien qué era la etnolingüística, pero yo me fui acercando cada vez más a la sociolingüística y con los años me fui convirtiendo cada vez más en lingüista y menos en antropóloga. En cierto momento la Universidad de Pennsylvania me ofreció un cargo en el departamento de lingüística, y así abandoné la antropología, aunque todavía llevo conmigo la formación antropológica, y es importante para mí.

\section{AM: ¿Y cuándo se conocieron?}

GS: En 1969, con mi anterior marido y colega, David Sankoff y con Henrietta Cedergren estábamos planeando la primera recolección de datos en Montreal (los primeros datos de los que hablé hoy en mi charla) y decidimos ir a Nueva York y preguntarle a Bill algunas cuestiones del diseño del trabajo. Ahí lo conocí. Nos encontramos varias veces en los años siguientes, y en 1973 me invitó a dar un curso con él ... ¿̇dónde era?

\section{William Labov (WL): Ann Arbor}

GS: Ann Arbor, Michigan. Yo ya había dado cursos como visitante en varias universidades como Stanford, Berkeley y alguna otra, así que cuando me llegó la oferta de la universidad de Pensilvania pensé que sería temporario también y me mudé. Estaba divorciada pero jamás pensé que sería definitivo. Bill y yo enseñábamos en el mismo departamento y después de un tiempo la relación cambió... y acá estamos. Nuestra hija menor tiene 26 años... iLlegamos bastante lejos!

\section{AM: ¿Pero ustedes nunca hicieron investigación juntos?}

GS: No, nunca. Bueno, ahora que hable Bill. Suficiente de mí.

WL: Yo llego a la lingüística desde otro campo, desde la química industrial.

\section{AM: ¿Su título de grado es en química?}

WL: No, mi formación de grado en Harvard era en inglés y filosofía, pero siempre me entusiasmó la ciencia. Cuando salí de la universidad no sabía bien qué hacer, hice diferentes cosas. En el laboratorio de una pequeña compañía de serigrafía usé mi conocimiento de química para hacer las fórmulas de las tintas para impresión: tinta para cartón, para remeras, para botellas, etc. Pero después de un tiempo, por más que era un buen trabajo y me gustaba mucho la gente con la que trabajaba, me di cuenta de que
2. Sankoff, Gillian. 1968. Social aspects of multilingualism in New Guinea. Doctoral dissertation. Mc.Gill University 
no me permitía responder las grandes preguntas que me preocupaban. Y para colmo, cuando encontraba algo interesante no podía decirlo porque era un secreto industrial...

\section{AM: Las fórmulas, claro...}

WL: Así aprendí que el mundo real está ahí afuera y listo para que lo descubran. A mí me gustaba mucho hablar con los trabajadores de la fábrica, la gente de la imprenta, los obreros, los camioneros. Hablábamos todo el tiempo, de todo y se me ocurrió que yo podía aplicar el mismo método que utilizaba en el laboratorio para analizar el lenguaje. En esa época ${ }^{3}$ el trabajo de la mayoría de los lingüistas era totalmente abstracto, encerrados en las bibliotecas y preguntándose a sí mismos si tal cosa podía decirse o no. Pensé que yo podía hacer algo mejor, que podía salir a ver si realmente esas cosas se decían o no, qué decía la gente y por qué para capitalizar lo que había aprendido en la industria. Y entonces apareció un invento nuevo, el grabador. Así que a los 30 años volví a la universidad a investigar sobre el lenguaje... hice mi doctorado con Uriel Weinreich, y pude desarrollar una lingüística empírica, basada en lo que la gente dice realmente, donde todo se puede testear con las técnicas experimentales del laboratorio. Y descubrí dos cosas. Primero, que cuando ponías un micrófono delante de una persona y le hacías preguntas obtenías una forma de habla diferente de la habitual y que si yo quería recolectar la forma de hablar habitual tenía que lograr que la gente pensara en lo que estaba diciendo y no en cómo lo estaba diciendo.

\section{AM: La paradoja del observador.}

WL: Sí. En segundo lugar, encontramos que la gente no dice dos veces las cosas exactamente igual, pero cuando queríamos describir eso no podíamos, no teníamos las herramientas necesarias, porque la lingüística no usaba números. Así empezamos a desarrollar el campo de la lingüística cuantitativa aplicando las mismas técnicas de otras ciencias, lo que luego se convirtió en una disciplina mayor que en la actualidad es el estudio del cambio lingüístico y la variación.

\section{AM: ¿Cómo se le ocurrieron los métodos de recolección de datos, como el estudio de los grandes almacenes?}

WL: Bueno, eso me llevó un día y medio de trabajo... Estaba trabajando en Low East side, en la caracterización de una comunidad, y la pregunta era qué tan característica era el habla de esa comunidad en relación con el habla de la ciudad de Nueva York como un todo. ¿Te acordás de Mi bella dama? Henry Higgins decía que podía identificar casi en qué cuadra de Londres vivía una persona por cómo hablaba. . ../../../.././acmen/Documents/ AHORA/SyS. Labov/Entrevista a Labov y Sankoff completa.doc - ftn4 Pero no es verdad, Nueva York, como París, Londres o Buenos Aires, son geográficamente una unidad, lo mismo que pasa en cada barrio. El estudio de las grandes tiendas fue un día de trabajo que me permitió recoger rápidamente muestras de habla de gente de toda la ciudad de Nueva York y ver si había algún patrón vinculado a la edad, la clase social o el género.

\section{GS: Pero Andrea te preguntó cómo se te ocurrió la idea...}

WL: Bueno, los lingüistas somos muy hábiles analizando datos pero no recogiéndolos, en cambio los psicólogos son muy ingeniosos para recolectar datos y han desarrollado muchas técnicas desde Pavlov en adelante. En lingüística hay que ser muy cuidadosos para recoger los datos, porque
3. Labov trabajó como químico industrial entre 1949 y 1961.
4. - Simply phonetics. The science of speech. That's my profession; also my hobby. You can spot an Irishman or a Yorkshireman by his brogue. I can place any man within six miles. I can place him within two miles in London. Sometimes within two streets. (Shaw, George Bernard. 1912. Pygmalion. http:// www.kkoworld.com/kitablar/Bernard_ Shaw_Secilmis_eserler_eng.pdf 
eso es lo que la gente va a recordar. Supongo que por eso se recuerda tanto el estudio de las grandes tiendas. Esa investigación se convirtió en una lectura obligada en muchas escuelas secundarias precisamente por la técnica de recolección.

GS: Pero quisiera decir que Bill siempre está pensando formas de descubrir algo. Lo llama pensar experimentos de campo. Cuando nuestros estudiantes empiezan con una nueva investigación y tienen que pensar cómo encontrar el fenómeno en el habla espontánea, el aparece con alguna idea. Por ejemplo, uno de mis estudiantes estaba estudiando un dialecto del sur de EEUU donde el diptongo /ai/ monoptonga en /a:/, por ejemplo en lugar de /faiv/ dicen /fa:v/ (five) y en lugar de /raid/ dicen /ra::d/(ride) o algo así. Bill se acerca a mi alumno y le dice “¿sabés cómo podés conseguir un montón de datos para eso? Cuando sean casi las cinco empezá a preguntarles a todos qué hora es... así vas a recoger un montón de datos”... No sé cómo se le ocurren esas ideas, pero siempre se le ocurren.

WL: Nosotros tenemos muchos experimentos de campo, como por ejemplo el siguiente. Después de hablar media hora de cualquier cosa que sea importante para el hablante, le decimos "hay cuatro maneras de decir / down/ (y las pronunciamos). ¿Cuál es la que usted usa?” Por supuesto, el hablante no contesta cuál es la que usa sino la que cree que debe usar, es decir, reflexiona sobre la norma, reflexiona hacia dónde se mueve conscientemente. Eso es muy importante porque muchos de los cambios que observamos en el inglés americano están por debajo del nivel de consciencia, pero la gente apenas se mueve en la dirección del cambio cuando se lo preguntás. Ahora, en psicología están más acostumbrados al uso de técnicas ingeniosas como los tests de pares ocultos ${ }^{5}$, por ejemplo, en los que escuchan a la misma persona hablando de dos maneras diferentes. Así desarrollamos algunas técnicas ingeniosas para descubrir las actitudes lingüísticas subyacentes de la gente hacia la lengua del hablante, hacia algunas variantes lingüísticas en particular a través del recurso de que la misma persona use las distintas formas. Ahora también estamos sacando provecho de las nuevas tecnologías de síntesis de voz. A medida que aparecen nuevas tecnologías también se van desarrollando nuevas técnicas de recolección y análisis que nos permiten hacer nuevas preguntas. Por ejemplo, el Atlas del inglés de Norteamérica se basa en tecnología inimaginable cuando empecé a recoger mis primeros datos ${ }^{6}$.

\section{AM: En relación al Atlas, ¿cuánto tardaron en recoger los datos y analizarlos?}

WL: En total nos llevó diez años, tardamos cinco años para recoger y analizar los datos y después cinco años más para publicarlo. El principal problema fue que observábamos que el cambio lingüístico se estaba dando tan rápidamente que con los métodos tradicionales de recolección y transcripción nos iba a llevar demasiado tiempo. No queríamos tener una distancia temporal muy amplia entre la primera entrevista y la última. Además la mayor parte del territorio nunca había sido analizada exahustivamente, así que decidimos utilizar las nuevas tecnologías e hicimos la recolección telefónica, lo que nos permitió llegar a todas las ciudades de Estados Unidos y Canada. Tardamos dos semanas en hacer el análisis espectral de cada hablante. Eso fue bastante exitoso en su momento, pero ahora ya tenemos otras técnicas y softwares que permiten hacer gran parte del reconocimiento de forma automática. Esas técnicas están transformando la disciplina... vamos a poder analizar 10000 muestras en una hora.
5. Matched guise tests.

6. Labov, William, Sharon Ash y Charles Boberg (2008). The Atlas of North American English. Phonetics, Phonology and Sound Change. Berlin, Boston: De Gruyter Mouton. El Atlas ofrece el primer relevamiento general de la pronunciación y del sistema vocálico de los dialectos de inglés de Estados Unidos y Canadá mostrando el cambio lingüístico activo en los noventa. Se basa en una encuesta telefónica de 762 hablantes que representan todas las zonas urbanas de Norteamérica. 
AM: En lugar de un hablante de dos semanas

WL: En lugar de 300 mediciones en dos semanas, 10000 mediciones en una hora. Eso es un cambio radical en la cantidad de datos que podemos analizar y va a cambiar totalmente la disciplina.

\section{AM: ¿Y ustedes están usando esas técnicas con muestras recogidas en internet, por ejemplo?}

WL: No, aplicamos estas nuevas tecnologías en el corpus de datos que venimos recogiendo desde 1973. Todos los años, una vez por año, un grupo de estudiantes, bueno, dos o tres grupos, van a cada lugar, se presentan, recogen los datos y luego los analizan. Y así hemos conformado un corpus muy grande que podemos analizar con estas nuevas tecnologías, y podemos hacer nuevas preguntas. Usamos esos datos para publicar un artículo que se llama Cien años de cambios de sonidos en Filadelfia ${ }^{7}$. Analizamos el habla de más de 300 hablantes que nacieron entre 1888 y 1992. El uso de las nuevas tecnologías nos da la oportunidad de hacer nuevas preguntas. Así nos encontramos con que algunos de los cambios han estado activos y se mantuvieron en la misma dirección durante los cien años, pero otros cambios que estaban activos e iban aumentando de pronto se han frenado y han retrocedido, y todo sucede más o menos al mismo tiempo, entre la gente que nació en 1950 y 1940. Y eso nos lleva a una nueva pregunta: ¿por qué? Porque en el mismo período nos encontramos con cambios en sentido contrario en varias ubicaciones diferentes. Los rasgos comunes con los dialectos del norte aceleraron su proceso de cambio, mientras que los rasgos comunes con los dialectos del sur retrocedieron. Y esto abre una nueva serie de preguntas que todavía no podemos responder ¿por qué? ¿por qué sucede al mismo tiempo la aceleración de ciertos cambios lingüísticos y el retroceso de otros?

GS: Andrea, yo quisiera agregar que afortunadamente el Atlas está disponible en línea gratuitamente en la página de la editorial. ${ }^{8}$ Eso es muy bueno.

AM. Es increíble la cantidad de cosas inimaginables hace unos años que ahora podemos hacer. ¿Existe un atlas lingüístico de Canadá?

GS: No, no. El inglés de Canadá está incluido en el Atlas lingüístico de Norteamérica, pero no hay ningún atlas completo que incluya el francés y las otras lenguas. Hay algunos proyectos por regiones, pero ningún atlas que reúna todas las regiones. Además casi todos quedaron truncos porque están hechos con métodos muy tradicionales que quedaron antiguos y que requieren demasiado tiempo para completarse.

WL: Charles Boberg, uno de los autores del Atlas, trabajó mucho sobre el inglés de Canadá.

GS: Canadá es un ambiente mucho menos propenso a la asimilación de los inmigrantes que los Estados Unidos, por eso hay más estudios sobre bilingüismo y multilingüismo. En Toronto, la ciudad más grande, hay muchas comunidades de inmigrantes que mantienen su lengua de origen, mucho más que en EEUU.

AM: ¿Siguen siendo comunidades separadas, sin interacción frecuente y sin bilingüismo?

GS: En Montreal hay un grado de bilingüismo mayor que el que solía haber, pero en Quebec, por ejemplo, nadie habla inglés. Montreal está en el oeste de Canadá, más cerca de la región angloparlante y hay más hablantes de inglés, pero son una minoría. Aun así, cada vez hay más
7. Labov, W. \& Rosenfelder, I. \& Fruehwald, J. (2013). One Hundred Years of Sound Change in Philadelphia: Linear Incrementation, Reversal, and Reanalysis. Language 89(1), 30-65. Linguistic Society of America. Retrieved October 7, 2018, from Project MUSE database.
8. En 2013 pude descargar los capítulos del Atlas de la página de la editorial De Gruyter, pero no acceder a los mapas interactivos. En la actualidad hay más restricciones para el acceso. http://www.degruyter.com/viewboo$\mathrm{ktoc} /$ product $/ 178229$

http://www.ling.upenn.edu/phono atlas/home.html

http://www.degruyter.com/ view/product/178229 
bilingües francés-inglés. Yo crecí en un entorno angloparlante, y cuando decidí, digamos, mudarme al francés, la gente estaba horrorizada, era algo tan inusual... Ahora ya no lo es tanto. Yo no podía soportar la idea de no poder hablar con todos, así que tuve que aprender francés.

\section{AM: ¿Y cómo aprendiste francés?}

GS: Bueno, en la escuela, a partir de tercer grado, todos aprendíamos. No era un programa muy exitoso, pero yo estaba muy motivada para aprender y usar el francés siempre que pudiera. Vivía en un vecindario atípico donde había tanto hablantes de inglés como de francés, y yo no desperdiciaba ninguna oportunidad de hablar con ellos. Ya de adolescente, por ejemplo, cuidaba niños de familias que hablaran francés

WL: El aspecto social del lenguaje es difícil de estudiar porque no es tan fácil calcularlo ni medirlo. La pregunta de por qué cambia la lengua ciertamente es muy amplia y necesitamos comprenderla mejor, pero si nos referimos a lo que antes llamábamos el problema de la actuación, es decir, por qué ahora, por qué en este lugar, también es muy misterioso y solo estamos adivinando...

AM: Entonces, si pensamos en la comunidad chomskiana, la comunidad lingüística idealizada, del todo homogénea... ¿̇habría cambio lingüístico?

WL: Bueno, la comunidad chomskiana idealizada no existe. La lengua de Chomsky está acá dentro (señalando su cabeza) mientras que para nosotros está ahí afuera, en la comunidad. La comunidad para nosotros los sociolingüistas es la unidad básica para la explicación. Bueno, no para todos, algunos sociolingüistas piensan que la unidad es el individuo, porque hablamos con individuos... el misterio más grande es cómo llegan los chicos a tener ese sentido de comunidad cuando aprenden el lenguaje... Los niños aprenden la lengua de sus padres pero de maneras que nos parecen mágicas abandonan totalmente los rasgos más idiosincrásicos de los padres para asumir otros.

GS: Bueno, no totalmente, pero en gran medida, sí.

WL: Es decir que los niños abandonan algunas de las características lingüísticas de sus padres para unirse a la comunidad, pero ¿qué tan grande es esa comunidad que el niño percibe? ¿Cómo la percibe? Los chicos van a la escuela y adoptan los rasgos de sus compañeros, pero también vemos que adoptan los rasgos de los chicos con los que están en conflicto, de los chicos con los que se pelean todo el tiempo... Así que la noción de comunidad que define el lenguaje es todavía elusiva pero sabemos que es real y que se constituye socialmente. No es, como dice Chomsky, una cuestión de libre albedrío. Podés decir lo que quieras, sí, pero hay consecuencias que pagar. Yo puedo hablar en chino, pero hay consecuencias si yo hablo en chino, ila gente que está conmigo no me va a entender! Es decir, como persona, como individuo, podemos desafiar las normas sociales, pero hay un precio que pagar por eso, que a veces es muy alto, como mostré en los estudios del habla de Nueva York. La gente no consigue el empleo que quiere si no habla como se espera en ese empleo.

AM: ¿Se puede decir que usted abrió ese debate cuando analizó la estructura del inglés no estándar de la comunidad afroamericana? Incluso contribuyó al debate sobre cómo llamar a la lengua... 
WL: Hay gente que cree que mi mayor contribución fue un trabajo que se llama The logic of non standard English ${ }^{9}$. No había mucha lingüística cuantitativa en ese trabajo, solo demostré que el habla de las comunidades afroamericanas es un sistema regular e intenté enfrentar un problema social por medio de la persuasión. Básicamente lo que encontré es que había reglas sistemáticas en lo que parecía una variación caótica. Hoy todavía seguimos intentando aplicar lo que encontramos en la preparación de materiales para la enseñanza de la lectura y la escritura. Escribí muchos programas, entre ellos uno gigante, en el que intenté aplicar todo mi conocimiento sobre los niños afroamericanos y su lenguaje. Siempre me interesó el tema profundamente, y pude juntar mi interés por las narrativas y por la enseñanza del inglés en la preparación de un material específico para estos chicos.

GS: Son dos materiales diferentes, para enseñar a leer y para apoyo escolar de chicos más grandes que tienen dificultades con la lectoescritura. Son materiales muy interesantes para el trabajo en la escuela, porque no solo están pensados lingüísticamente sino también culturalmente. Las cosas que leen los chicos están pensadas para ellos, y se basan en sus propias experiencias. ${ }^{10}$

WL: Las historias se basan en cosas que algunos chicos me contaron realmente e incluyen conflictos y reflexiones sobre esas situaciones conflictivas. Por ejemplo, un chico que va a la escuela y en el camino ayuda a una chica a buscar su gato entre las plantas, se engancha la ropa y se le rompe. Cuando llega a la escuela se queda con la campera puesta, la maestra le dice "Sacate el saco", pero él dice que no, y después de insistir y de negarse varias veces, la maestra lo castiga. ¿Y por qué no dijo nada para evitar el castigo? Porque le daba vergüenza. ${ }^{11}$ Son contradicciones habituales en las que entramos continuamente en nuestra vida social cuando intentamos ajustarnos a las normas que creemos que debemos seguir.

GS: Bueno, ves cómo le gustan a Bill las narrativas... Él mismo escribió las historias de los libros para niños. Por ejemplo, hay una que se llama "I hate that hat", en la que un chico es obligado por su madre a llevar un gorro que no le gusta. Las actividades no solo reflexionan sobre cómo cambia la pronunciación y la ortografía sino también sobre el sentimiento de injusticia que nos provoca hacer algo que no queremos.

\section{Julián Ezquerra (JE): ¿Podemos decir que hay simultáneamente un} objetivo lingüístico y un objetivo social?

WL: El principal objetivo es que los niños se sientan parte del sistema educativo, que leer y escribir también es para ellos, que no sientan que el sistema lo expulsa. El estudio del lenguaje en la vida social nos dice cómo y por qué el lenguaje está cambiando, pero también nos permite conocer mejor a la gente. Y como lingüistas, podemos ayudar a la gente a cambiar su lengua en la dirección que desean. Los conocimientos que obtuvimos como lingüistas pueden ser aplicados en la educación, pero también en otros órdenes de la vida también, por ejemplo en la investigación forense. En mi charla sobre Beatriz Lavandera intenté mostrar cómo se pueden enfrentar problemas sociales inmediatos con el análisis del discurso. Lavandera mostró claramente algunas de las dificultades que tiene analizar la forma y el significado simultáneamente, y se necesita cierto grado de ingenio.
9. Labov, W. 1969. The logic of non standard English. En Alatis, James (ed). 2oth Annual Round Table. GURT. Georgetown University Round Tables. Washington, GUP.

10. Se trata de dos obras diferentes, The reading road y Portals. La primera está disponible a través de la página de The reading inniative de la Universidad de Pennsylvania, en el siguiente vínculo: http://www.ling.upenn.edu/ pri/readingroad/downloadall.html

11. La historia está en Portals. Rea World Reading Bounce, publicada por Houghton Mifflin Harcourt como un programa de apoyo a la lectura en la escuela. 
AM: Gillian, en tu conferencia mostraste una versión diferente de cambio lingüístico, el cambio a lo largo de la vida de una persona ${ }^{12}$. GS: Sí, el cambio lingüístico a lo largo de la vida es el tema de mi investigación actual. La primera encuesta de Montreal fue realizada en $1971^{13}$, y utilizó un método de muestreo aleatorio estratificado para lograr una muestra de 120 hablantes controlando la edad, el sexo y la clase social. En 1984 se localizaron 60 de los 120 entrevistados y se los volvió a entrevistar, y en 1995 se entrevistó nuevamente a 12 de esos 60. De esta manera fue posible obtener un corpus que garantiza la interpretación del cambio lingüístico como cambio ocurrido a lo largo de la vida de la persona. Tuve mucha suerte de que mis estudiantes quisieran llevar adelante este estudio y que encontraran tantos hablantes de la muestra original.

\section{JE: ¿Eran todos canadienses de habla francesa?}

GS: Sí, todos canadienses de habla francesa que hubieran vivido en Montreal por lo menos desde los 5 años. En Montreal hay anglófonos, francófonos y ¿sabés cómo se les dice a los inmigrantes que no hablan ni inglés ni francés? "Alófonos". A ellos no los estudiamos.

WL: Quisiera agregar que el estudio original de Gillian de Montreal es el mejor diseño de una investigación sociolingüística que se haya hecho hasta ahora. Y es la base sobre la que nuestros estudiantes, milagrosamente, lograron encontrar a 60 de esos hablantes.

AM: Eso le iba a preguntar, ¿cómo fue que lograron retomar ese estudio y encontrar a los hablantes? ¿Una cuestión de suerte o de diseño?

GS: Yo ya estaba en EEUU y para ese momento mis antiguos alumnos eran profesores e investigadores en Laval, Quebec etc. Y decidieron llevar adelante el estudio, y encontraron a 60 de los 120 hablantes del estudio original. Eso fue mucha suerte.

WL: Ese estudio abrió la puerta para un nuevo campo de estudio en sí mismo, el cambio lingüístico a lo largo de la vida ${ }^{14}$.

GS: Efectivamente, muchos sociolingüistas que hicieron sus estudios en los 60 y 70 están volviendo a las comunidades que estudiaron para analizar el cambio lingüístico en los mismos hablantes de la misma manera que lo hice yo hoy.

AM: ¿Cuál es su opinión respecto de la hipótesis del período crítico para la adquisición de la lengua?

GS: Es la pregunta más interesante. Sí, yo soy una creyente en el período crítico, hay ciertas cosas que no se modifican. Algunos consideran que porque en un tercio de nuestra muestra hay algún tipo de cambio después de los 15 años, eso nos da pie para falsear la hipótesis del período crítico, pero no, no. Hay cosas que no se modifican. Si no, no habría tantas dificultades para los migrantes adultos, podrían aprender la lengua sin problema. Que haya cambios no significa que no haya un límite, solo que ese límite no es taxativo, es una curva, y como en todos los fenómenos de la vida hay gente más maleable y gente menos flexible.

AM: Quisiera volver un momento a los 60 y preguntarles por algo que todos hemos estudiado: la revolución chomskiana, las guerras lingüísticas, el nacimiento de la sociolingüística cuantitativa y la
12. Presentó datos recolectados entre los años setenta y la actualidad y reflexionó en profundidad sobre cuestiones críticas de la interpretación de las variables lingüísticas en relación con la variable edad: cuándo es posible interpretar que las correlaciones observadas se deben a un cambio lingüístico en marcha, y cuándo se trata simplemente de cambios lingüísticos naturales que el ser humano sufre a lo largo de su vida que no llevan a un cambio en la lengua de la comunidad. 13. El diseño original se encuentra en Sankoff, Gillian, \& Cedergren, Henrietta J. (1972). Sociolinguistic research on French in Montreal. Language in Society 1:173-174. 
gramática universal, y el surgimiento de la gran división entre la sociolingüística y la lingüística generativa. Desde nuestro lugar, los 60 fueron un período en el que los lingüistas estaban en guerra unos con otros. ¿Cómo fue la pelea Chomsky - Labov?

WL: Jajaja, no, en realidad fue al revés. Como dije antes, la lingüística de los 50 y los 60 era muy inadecuada para estudiar el lenguaje en la vida cotidiana. Mi profesor, Uriel Weinreich, fue el primero en ver la importancia del programa generativo para el análisis lingüístico, y desde entonces yo trabajé siempre dentro del marco generativo. Las contribuciones de Chomsky son importantes fundamentos. Pero otra manera de ver las diferencias es esta: a Chomsky le interesa la gramática universal, que es el resultado de una mutación que ocurrió hace...digamos, quizás unos treinta o cuarenta mil años atrás, y que desde entonces no ha cambiado. Es algo que no cambia con el tiempo. A mí me interesa, en cambio, todo lo que podría cambiar, lo que cambia, que es una parte bastante grande del lenguaje. Yo estoy muy abierto a todas las sugerencias que se han hecho acerca de las características lingüísticas que pertenecen a la gramática universal, como la recursividad, por ejemplo, aunque en la sociolingüística y en la lingüística histórica nos preocupen las preguntas por el origen del lenguaje como un todo... Sin embargo, cuanto más analizamos los fenómenos lingüísticos, más respeto tenemos por las generalizaciones más exitosas que recurren a los conceptos de estructuras programadas en el cerebro fuera del nivel de la conciencia. Esa es la contribución de Chomsky más importante. Sin importar si el modelo generativo persista en su forma actual o no, la gramática universal tiene que permitir que un chico aprenda cualquier lengua.

GS: Es interesante la pregunta... por un lado creo que las guerras lingüísticas se dieron sobre todo porque en los congresos la gente se peleaba públicamente a los gritos por sus diferencias teóricas. Me parece que el conflicto se dio sobre todo en el campo de la lingüística teórica... por otro lado creo que al principio los sociolingüistas malentendimos lo que Chomsky quería decir con lo del hablante oyente ideal y la comunidad idealizada. Entendimos que estaba hablando de la comunidad real, cuando en realidad estaba simplemente haciendo una abstracción y hablando de otra cosa diferente que en ese momento era nueva. Estudiamos cosas diferentes y nos interesan cosas diferentes. Hoy nadie discute eso, y nosotros mismos utilizamos los insights de la lingüística teórica para entender la estructura lingüística.

WL: Chomsky, entre otras cosas, le ha dicho a tres generaciones de estudiantes que la lingüística no tiene aplicaciones prácticas. Yo no estoy de acuerdo con eso. Pero también ha hecho hincapié en que, cualquiera sea la lengua, para que sea una lengua posible tiene que poder ser aprendida por un niño. Y también en que el conocimiento de la sintaxis no es un conocimiento teórico abstracto sino una realidad cognitiva que tiene que permitir la adquisición. Eso es muy importante.

AM. ¿Siguen pensando en la regla variable como una posibilidad teórica dentro del programa generativo?

WL: Bueno, eso ha cambiado mucho, hay mucha gente que está trabajando en gramáticas probabilísticas. Muchos de mis estudiantes ponen el foco en tratar de descubrir qué fenómenos variables pertenecen realmente a la gramática y cuáles no. Por ejemplo, hemos encontrado que los niños tienen frecuencias de variación muy cercanas a las de sus padres, y probablemente eso sea un fenómeno ajeno a la gramática. El esfuerzo no es llegar a decir 
que todo es cuantitativo y numérico, sino en qué consiste el conocimiento gramatical y por qué los chicos copian con tanta precisión las frecuencias de variación de sus padres. La respuesta a la pregunta, entonces, es si los números y la variación son parte de la gramática o no. Las reglas variables son una respuesta que se me ocurrió en ese momento haciendo uso de la noción de regla que se estaba utilizando, pero hoy la pregunta sigue siendo cómo el comportamiento variable encuentra su lugar en la estructura de la lengua. Hay un cierto número de gramáticos generativos que están dispuestos a recurrir a algunos conceptos probabilísticos, pero yo no diría que toda la disciplina en su conjunto.

Lo que digo es que la gente que basa sus estudios en la cuantificación del discurso cotidiano es todavía una parte menor. Trabajar con corpus de datos recogidos de grabaciones a gran escala, es decir, el enfoque sociolingüístico es solo una manera de acercarse al fenómeno lingüístico y no es el más común. Dicho de otra manera, la pregunta es ¿qué es lo que este acercamiento a la variación y el cambio tiene para ofrecerle al estudio de las lenguas poco estudiadas, cuando hay que describir una lengua por primera vez?

\section{AM: Eso me recuerda la pregunta que Beatriz Lavandera siempre nos hacía: ¿qué es lo que esta investigación tiene para decir sobre el lenguaje?}

WL: En este momento la tesis que acaba de terminar uno de mis estudiantes trata sobre la naturaleza del cambio lingüístico, y argumenta que los efectos fonológicos ocurren con más frecuencia tempranamente y no más adelante en el proceso de cambio. Esa es una contribución muy importante a nuestro conocimiento respecto de cómo la fonología interactúa con la fonética. Otra tesis trata un tema que fue bien desarrollado por lingüistas brasileños, la persistencia, es decir la tendencia de una variable a aparecer con más frecuencia cuando es precedida por la misma forma. El trabajo de Shana Poplack lo mostró en el español, la variante cero es precedida con más frecuencia por la variante cero, y la -s, por la -s. Por ejemplo, en "las cosas bonitas", es más frecuente encontrar la falta de -s o la aparición de -s en las tres formas que solo en el determinante. Esto contradice las expectativas que se basan en la teoría de la información, según la cual una vez que tenés la -s en el determinante ya es suficiente para conservar la información y por lo tanto sería esperable mayor frecuencia de -s -cero- cero ${ }^{15}$.

\section{AM: No sé si estoy segura de entender... ¿El mismo fenómeno que estudió Poplack se da en portugués?}

WL: Sí, exacto. Los sonidos finales son una señal que tiende a desaparecer, y sin embargo, la tendencia contraria a lo esperado en español es que las marcas del plural se repiten. Es lo que se llama persistencia, algo similar a lo que en psicología es el efecto priming, la tendencia a que una opción sea seguida por la misma opción.

El fenómeno de persistencia está muy extendido y tiene un montón de consecuencias teóricas: una vez que el sistema produce un resultado, es más fácil producir el mismo resultado. Y esto, por supuesto, interactúa con factores sociolingüísticos, por lo que nos encontramos que el fenómeno de persistencia es más notable cuando el elemento tiene una baja frecuencia. Cuando la variante es poco frecuente, es más persistente. Eso a veces se llama el efecto de frecuencia inversa. Es decir, no hay dudas de que los estudios de la distribución y variación cuantitativa tienen consecuencias teóricas importantes. Este es un ejemplo.
15. Poplack, S. (1980). The notion of the plural in Puerto Rican Spanish: Competing constraints on (s) deletion. Locating Language in Time and Space, 55-66. 
Yo no estoy, en este momento, entre los arquitectos de la estructura lingüística. Acepto el hecho, a través del trabajo de gente como Anthony Kroch, que trabaja conmigo, que la mejor conjetura es que esa arquitectura esté basada en el marco generativo. Recién estuvimos hablando de algunas modificaciones en ese enfoque, pero mi preocupación es el problema del cambio y la variación lingüística. El cambio lingüístico involucra preguntas sobre las posibles restricciones al cambio, cuál es el mecanismo del cambio, cómo se inscribe el cambio en la estructura lingüística, cómo se inscribe el cambio en la estructura social, cómo se evalúa el cambio, y por qué un cambio particular ocurre en un cierto lugar y tiempo. Esas son las preguntas teóricas centrales que nos hemos hecho, y hemos constituido una especie de zona propia a través del estudio de la variación lingüística en la sociedad.

La arquitectura formal del lenguaje nos abre un amplio conjunto de preguntas, y a través del cambio lingüístico podemos, quizás, responder algunas, pero no es el foco central de nuestro trabajo. El foco central para nosotros todavía está en cuál es la naturaleza del cambio en los usos que los humanos hacen de su lengua, que llevan a una continua renovación, a nuevas formas de diferenciar las lenguas unas de otras. Esas son las preguntas teóricas fundamentales que nos interesan.

AM: Bueno, imuchísimas gracias a ambos! 